\title{
Flavanol-anthocyanin condensed pigments in plant extracts
}

\author{
Ana M. González-Paramás, Fátima Lopes da Silva ${ }^{1}$, Pilar Martín-López, \\ Glenda Macz-Pop, Susana González-Manzano, Cristina Alcalde-Eon, \\ José Joaquin Pérez-Alonso, M. Teresa Escribano-Bailón, \\ Julián C. Rivas-Gonzalo, Celestino Santos-Buelga *
}

Unidad de Nutrición y Bromatología, Facultad de Farmacia, Campus Miguel de Unamuno s/n, Universidad de Salamanca, E-37007 Salamanca, Spain Received 2 August 2004; received in revised form 25 November 2004; accepted 25 November 2004

\begin{abstract}
Pigments resulting from the direct condensation of anthocyanins and flavanols are usually associated with reactions taking place during processing and storage of plant-derived foods and beverages and have been particularly studied in aged red wines. In this paper, small amounts of flavanol-anthocyanin condensed pigments are found in different plant extracts. Structures are suggested for 10 such condensed pigments detected in extracts of strawberry, runner beans, purple corn and grape skins, based on their $\mathrm{MS}^{n}$ fragmentation patterns, following analyses by electrospray tandem mass spectrometry. All of them correspond to dimers containing a flavan-3-ol [either (epi)afzelechin, (epi)catechin or (epi)gallocatechin] as the upper unit carbon-carbon linked to a lower anthocyanin unit consisting of different delphinidin, cyanidin, pelargonidin, peonidin or malvidin derivatives. The detection of these pigments in plant extracts may suggest that they are natural pigments and not products exclusively formed during storage and ageing of processed foods and beverages, as was previously assumed.
\end{abstract}

(C) 2004 Elsevier Ltd. All rights reserved.

Keywords: Flavanol-anthocyanin; Condensed pigments; Strawberry; Runner bean; Purple corn; Grape skin

\section{Introduction}

Anthocyanin-derived pigments constitute a group of compounds mostly associated with anthocyanin transformations taking place during the maturation and ageing of red wines. Basically, three types of anthocyanin-derived pigments have been described: ethyl-linked compounds resulting from acetaldehyde-mediated condensation between anthocyanins and flavanols, 4-substituted anthocyanin derivatives containing additional pyrano rings in the flavylium nucleus (i.e., pyranoantho-

\footnotetext{
* Corresponding author. Tel.: +34 923 294537; fax: +34 923294515.

E-mail address: csb@usal.es (C. Santos-Buelga).

${ }^{1}$ Present address: Escola Superior Agrária de Bragança, Campus de Santa Apolónia, P-5301-855 Bragança, Portugal
}

cyanins), and products from the direct condensation between anthocyanins and another flavonoid moiety, namely a flavanol.

Pyranoanthocyanins (Bakker et al., 1997; Bakker \& Timberlake, 1997; Francia-Aricha, Guerra, RivasGonzalo, \& Santos-Buelga, 1997; Fulcrand, Cameira dos Santos, Sarni-Manchado, Cheynier, \& Bonvin, 1996) and pigments resulting from acetaldehydemediated condensation (Bakker, Picinelli, \& Bridle, 1993; Dallas, Ricardo-da-Silva, \& Laureano, 1996; Francia-Aricha et al., 1997; Rivas-Gonzalo, BravoHaro, \& Santos-Buelga, 1995; Roggero, Coen, Archier, \& Rocheville-Divorne, 1987; Timberlake \& Bridle, 1976) have been extensively studied in model systems and their presence is also well documented in matured and aged red wines (Mateus, Pascual-Teresa, Rivas-Gonzalo, Santos-Buelga, \& de Freitas, 2002; Mateus, Silva, 
Santos-Buelga, Rivas-Gonzalo, \& de Freitas, 2002; Vivar-Quintana, Santos-Buelga, Francia-Aricha, \& Rivas-Gonzalo, 1999; Vivar-Quintana, Santos-Buelga, Rivas-Gonzalo, 2002). Pigments from the anthocyanin-flavanol condensation involving aldehydes other than acetaldehyde have also been described (Es-Safi, Cheynier, \& Moutounet, 2002; Pissarra, Mateus, Rivas-Gonzalo, Santos-Buelga, \& De Freitas, 2003; Pissarra, Lourenço, et al., 2004).

Fewer studies exist about pigments from the direct condensation between anthocyanins and flavanols. Their structures and contribution to the colour in ageing red wines were hypothesized in the late 1960s (Jurd, 1967, 1969; Jurd \& Somers, 1970; Somers, 1971). Two possible pathways were suggested for their formation: (1) nucleophilic addition of the hemiketal form of an anthocyanin through their C-8 or C- 6 positions at C-4 of a carbocation resulting from the cleavage of the interflavanic linkage of a flavanol oligomer; further conversion of the hemiketal form of the anthocyanin to the corresponding flavylium would yield $\mathrm{F}-\mathrm{A}^{+}$adducts; (2) electrophilic substitution of the anthocyanin flavylium form (C-4) by a flavanol (C-8 or C-6) to yield an A-F dimer, where the anthocyanin moiety would be in the flavene form; this latter could oxidize to the corresponding flavylium form $\left(\mathrm{A}^{+}-\mathrm{F}\right.$ adducts) or rearrange, either to a yellow xanthylium ion (Jurd \& Somers, 1970; Liao, Cai, \& Haslam, 1992; Santos-Buelga, Bravo-Haro, \& Rivas-Gonzalo, 1995) or to a doubly-linked A-F colourless structure containing an A-type interflavonoid bond (Bishop \& Nagel, 1984; Remy-Tanneau, Le Guernevé, Meudec, \& Cheynier, 2003). Detection, in red wines, of pigments showing molecular ions corresponding to either $\mathrm{A}^{+}-\mathrm{F}$ or $\mathrm{F}-\mathrm{A}^{+}$adducts was first achieved by Vivar-Quintana et al. (1999) using LC-MS and further confirmed by Salas et al. (2004) who also found evidence in favour of the $\mathrm{F}-\mathrm{A}^{+}$structure. The occurrence, in red wine, of doubly-linked A-F colourless dimers was also demonstrated by Cheynier's group (Remy, Fulcrand, Labarbe, Cheynier, \& Moutounet, 2000).

Recent evidence shows that certain anthocyanin-derived pigments occur, not only in red wines, but also (a)

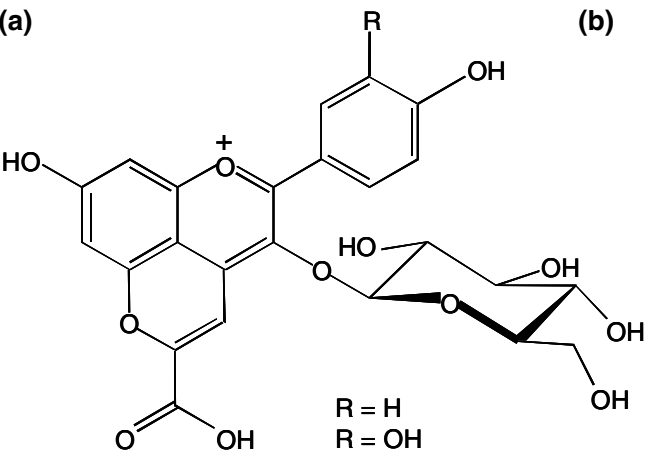

(b)

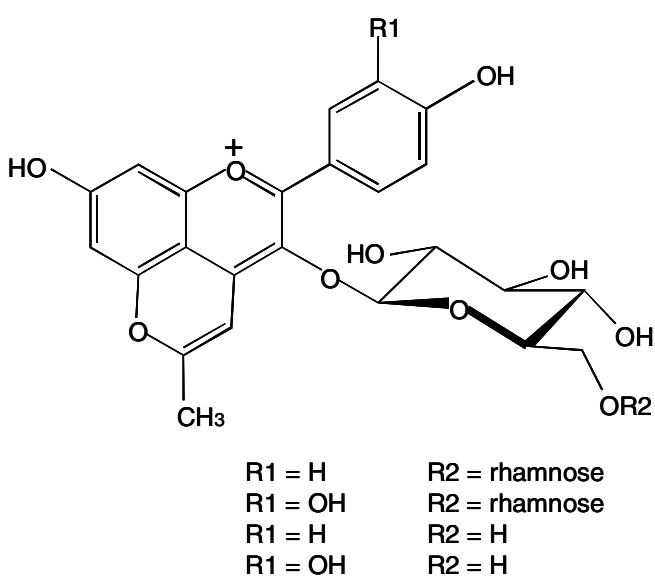

(c)<smiles></smiles>

(d)

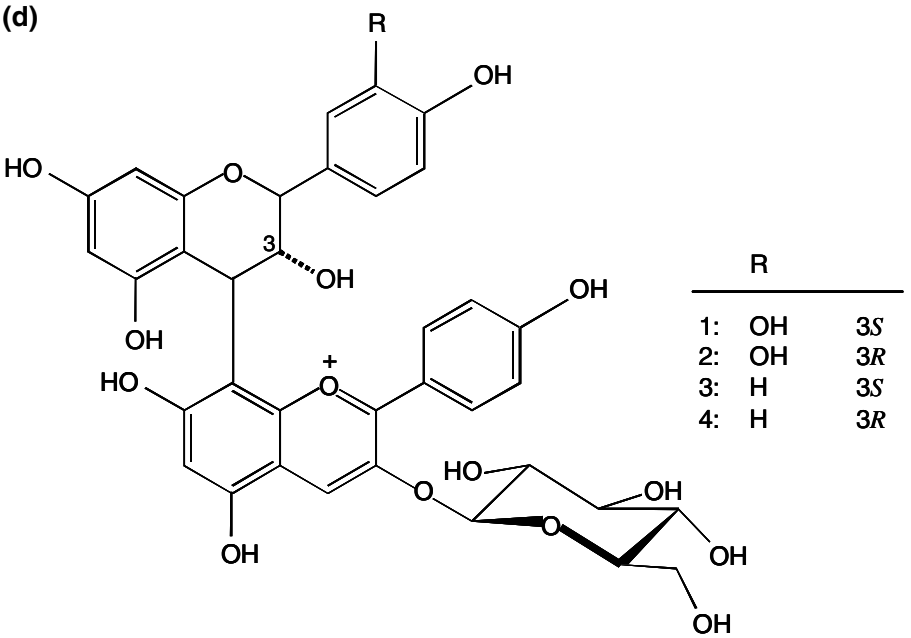

Fig. 1. Anthocyanin-derived pigments isolated from: (a) red onion (Fossen and Andersen, 2003) and strawberry (Andersen et al., 2004); (b) blackcurrant seeds (Lu et al., 2000); (c) petals of Rosa hybrida (Fukui et al., 2002); (d) strawberry (Fossen et al., 2004). 
in small amounts in plants. Thus, 5-carboxypyranocyanidin and 5-carboxypyranopelargonidin derivatives (Fig. 1(a)) have been obtained from red onion (Fossen \& Andersen, 2003) and strawberries (Andersen, Fossen, Torskangerpoll, Fossen, \& Hauge, 2004), and 5-methylpyranocyanidin and 5-methylpyranodelphinidin derivatives (Fig. 1(b)) from blackcurrant seeds (Lu, Sun, \& Foo, 2000). Also, a related 4-substituted cyanidin pigment containing two additional heterocycles attached to the anthocyanidin skeleton (Fig. 1(c)), derived from the $\mathrm{C}-\mathrm{C}$ condensation between gallic acid and the anthocyanin, have been extracted from petals of Rosa hybrida (Fukui, Kusumi, Masuda, Iwashita, \& Nomoto, 2002). Quite recently, four purple anthocyanin-flavanol complexes have also been found in strawberry extracts (Fragaria ananassa) and their structures elucidated by NMR, showing that they consist of pelargonidin 3-glucoside $\mathrm{C}-\mathrm{C}$ linked to (epi)catechin and (epi)afzelechin moieties (Fig. 1(d)) with an $\mathrm{F}-\mathrm{A}^{+}$structure (Fossen, Rayyan, \& Andersen, 2004). In the present work, further evidence indicates this kind of pigment in different plant extracts.

\section{Materials and methods}

\subsection{Sample preparation}

\subsubsection{Strawberries}

Strawberry samples (Fragaria x ananassa cv Camarosa) were homogenized in $0.1 \% \mathrm{HCl}$ in methanol and later filtered through a Büchner funnel under vacuum. The solid residue was exhaustively washed with methanol and the filtrates obtained were centrifuged. After addition of water, the supernatant was concentrated under vacuum to total evaporation of the methanol and the aqueous extract obtained was washed with $n$-hexane to remove liposoluble substances. An aliquot of the aqueous phase was deposited onto a C-18 SepPak $^{\circledR}$ Vac 3 cc cartridge (waters); sugars were removed by passing through $15 \mathrm{ml}$ of water and anthocyanin pigments further eluted with $5 \mathrm{ml}$ of $\mathrm{MeOH}: 0.1 \%$ TFA (95:5). The methanolic extract was concentrated under vacuum, redissolved in $2 \mathrm{ml}$ of ultrapure water and filtered through a $0.45-\mu \mathrm{m}$ membrane filter for High pressure liquid chromatography (HPLC) analysis.

\subsubsection{Beans}

The seed coat of scarlet red runner beans (Phaseolus coccineus) was manually separated and ground to obtain an homogeneous powder. Anthocyanin extraction was carried out by successive macerations in methanol containing $5 \%$ of $1 \mathrm{~N} \mathrm{HCl}$ in an ultrasonic bath. The methanol phases were pooled and centrifuged and the supernatant was concentrated under vacuum to total evaporation of the methanol. The aqueous extract ob- tained was washed with $n$-hexane to remove liposoluble substances and further purified using a C-18 SepPak cartridge, as described for strawberry anthocyanins.

\subsubsection{Purple corn}

A commercial anthocyanin-rich powder, obtained after drying of purple corn cob (cv. Morado) by a Peruvian company, was used (de Pascual-Teresa, SantosBuelga, \& Rivas-Gonzalo, 2002). The corn powder was dissolved in $0.01 \mathrm{M} \mathrm{HCl}$ and filtered through a $0.45-\mu \mathrm{m}$ membrane filter (Millipore, Bedford, Mass., USA) previous to injection in the HPLC system.

\subsubsection{Grape skins}

Pigment extraction was carried out as described in Heredia, Francia-Aricha, Rivas-Gonzalo, Vicario, and Santos-Buelga (1998). Briefly, grape skins (Vitis vinifera cv. Tempranillo) were macerated in methanol containing $5 \%$ of $1 \mathrm{~N} \mathrm{HCl}$; the methanol extract was evaporated under vacuum and the aqueous phase obtained washed with $n$-hexane to remove liposoluble substances. The aqueous extract was deposited onto a column loaded with a mixed stationary phase composed of $20 \%$ Polyclar AT and 80\% Kieselgel 60; sugars and acids were eliminated by exhaustive washing with water and anthocyanin pigments further eluted with $0.1 \% \mathrm{HCl}$ in methanol. The eluate was concentrated under vacuum, redissolved in water and filtered through a $0.45-\mu \mathrm{m}$ membrane filter for HPLC analysis.

\section{2. $H P L C-D A D-M S$ analysis}

HPLC analyses were performed in a Hewlett-Packard 1100 series liquid chromatograph, using the method described by Lopes da Silva, de Pascual-Teresa, RivasGonzalo, and Santos-Buelga (2002). Separation was achieved on a $5-\mu \mathrm{m}$ AQUA ${ }^{\circledR} \mathrm{C} 18150 \times 4.6 \mathrm{~mm}$ column (Phenomenex ${ }^{\circledR}$, Torrance, CA) thermostatted at $35^{\circ} \mathrm{C}$. Solvents used were: (A) $0.1 \%$ trifluoroacetic acid (TFA) in water, and (B) gradient-grade acetonitrile, establishing the following gradient: isocratic $10 \% \mathrm{~B}$ for $5 \mathrm{~min}, 10-15 \%$ B over $15 \mathrm{~min}$, isocratic $15 \%$ B for 5 min, $15-18 \%$ B over $5 \mathrm{~min}$, and $18-35 \%$ B over 20 min, using a flow rate of $0.5 \mathrm{ml} \mathrm{min}{ }^{-1}$. Double on-line detection was carried out in a photodiode spectrophotometer, using $520 \mathrm{~nm}$ as the preferred wavelength, and in a mass spectrometer connected to the HPLC system via the UV cell outlet.

The mass spectrometer was a Finnigan LCQ (San Jose, CA) equipped with an ESI source and an ion trap mass analyser, which were controlled by the LCQ navigator software. Both the auxiliary and the sheath gas were a mixture of nitrogen and helium at flow rates of 6 and $1.21 \mathrm{~min}^{-1}$, respectively. The capillary voltage was $4 \mathrm{~V}$ and the capillary temperature $195^{\circ} \mathrm{C}$. Spectra were recorded in positive ion mode between 150 and 
$1500 \mathrm{~m} / \mathrm{z}$. The MS detector was programmed to perform a series of three consecutive scans: a full scan, an MSMS scan of the most abundant ion in the first scan and $\mathrm{MS}^{3}$ of the most abundant ion in the $\mathrm{MS}^{2}$, using a normalised collision energy of $45 \%$.

\section{Results and discussion}

Fig. 2 shows chromatograms of the anthocyanin profiles of the plant extracts analyzed. Together with the major peaks corresponding to anthocyanins identified elsewhere (de Pascual-Teresa et al., 2002; Hebrero, Santos-Buelga, \& Rivas-Gonzalo, 1988; Lopes da Silva et al., 2002; Macz-Pop, Rivas-Gonzalo, Pérez-Alonso, \& González-Paramás, 2005) the presence of small peaks, showing UV-Vis spectra similar to anthocyanins, were also observed in the first part of the chromatograms. Molecular ions of those peaks obtained with the mass detector (Table 1) showed them to be anthocyanin-flavanol dimers, similar to those previously detected in red wines (Salas et al., 2004; Vivar-Quintana et al., 1999) and recently characterised by Fossen et al. (2004) in strawberry extracts.

UV-Vis spectra of compounds St1-St4, detected in strawberry extracts, were similar to those observed by Fossen et al. (2004) for the flavanol-pelargonidin pigments identified by them. They show $\lambda_{\max }$ in the visible
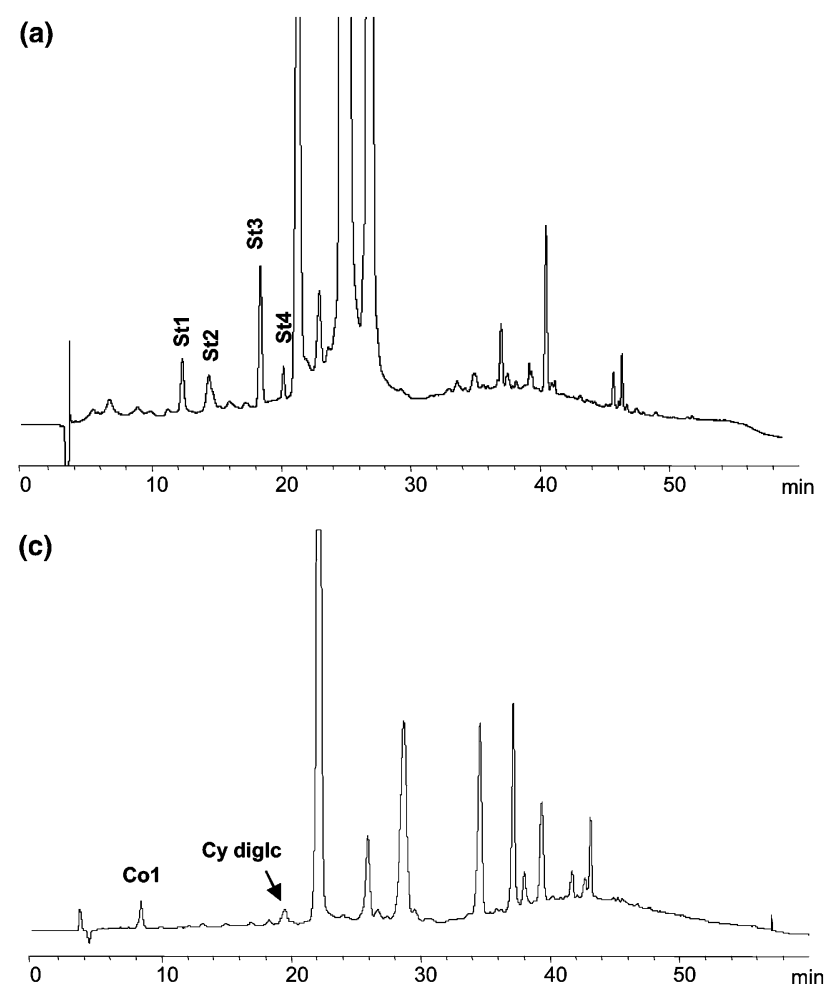

region at 515-518 $\mathrm{nm}$ (Table 1), bathochromically shifted with regard to that of the precursor anthocyanin (pelargonidin 3-glucoside, $\lambda_{\text {vis-max }}$ at $502 \mathrm{~nm}$ ). They also present increased absorption at $430-440 \mathrm{~nm}$ and, subsequently, an increased $A_{440 \mathrm{~nm}} / A_{\lambda_{\text {vis-max }}}$ ratio. Similar spectral differences in relation to the parent anthocyanin have been found for the condensed pigments detected in the different plants analysed in this work.

Peaks St1 and St2 possessed an identical molecular ion $[\mathrm{M}]^{+}$at $\mathrm{m} / \mathrm{z} 721$ and similar fragmentation patterns. Fig. 3 shows the $\mathrm{MS}^{2}$ and $\mathrm{MS}^{3}$ spectra of pigment St1 together with the fragmentation scheme. The $\mathrm{MS}^{2}$ fragment at $\mathrm{m} / \mathrm{z} 559$ ( $-162 \mathrm{amu}$ ) corresponds to the loss of a glucose moiety. $\mathrm{MS}^{3}$ led to fragment ions at $\mathrm{m} / \mathrm{z} 541$ $\left([\mathrm{M}-18]^{+}\right.$, loss of water $), 407\left([\mathrm{M}-152]^{+}\right.$, retro Diels-Alder fission of an (epi)catechin moiety), 313 $\left([\mathrm{M}-246]^{+}\right.$, partial loss of an (epi)catechin unit), 433 ([M - 126 $]^{+}$, loss of $\mathrm{C}_{6} \mathrm{H}_{6} \mathrm{O}_{3}$-A-ring-), and 271 ([M -288$]^{+}$, loss of an (epi)catechin unit). As was noticed by Friedrich, Eberhardt, and Galensa (2000) for the fragmentation of proanthocyanidin dimers and also observed by Salas et al. (2004) for $\mathrm{F}-\mathrm{A}^{+}$adducts found in red wines, the loss of a $\mathrm{C}_{6} \mathrm{H}_{6} \mathrm{O}_{3}$ fragment is characteristic of the upper flavanol unit. Furthermore, the loss of $288 \mathrm{amu}$ is expected when (epi)catechin constitutes the upper unit (but $-290 \mathrm{amu}$ when it is the terminal one). Therefore, the production of fragments at $\mathrm{m} / \mathrm{z}$ 433 and 271 reveals that the flavanol moiety (catechin
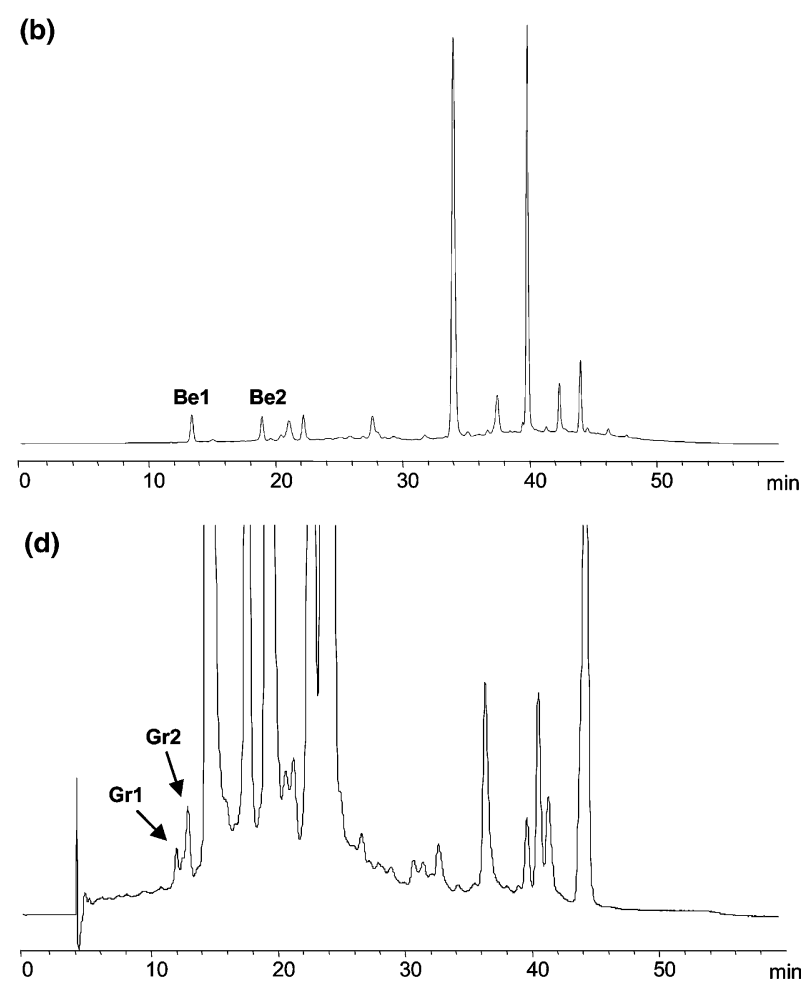

Fig. 2. HPLC chromatograms recorded at $520 \mathrm{~nm}$ of the different plant extracts: (a) strawberry; (b) runner beans; (c) purple corn; (d) grape skins. Peaks indicated correspond to flavanol-anthocyanin condensed pigments. 
Table 1

UV-Vis absorption maxima obtained on-line with the diode array detector and molecular ions of the flavanol-anthocyanin condensed pigments detected in different plant samples

\begin{tabular}{lll}
\hline Compound & $\lambda_{\text {vis-max }}(\mathrm{nm})$ & {$[\mathrm{M}]^{+}(\mathrm{m} / \mathrm{z})$} \\
\hline St1 & 515,430 & 721 \\
St2 & 517,436 & 721 \\
St3 & 515,430 & 705 \\
St4 & 518,433 & 851 \\
St5 & n.a. & 705 \\
Co1 & 528 & 899 \\
Be1 & 536 & 607 \\
Be2 & 532 & 737 \\
Gr1 & 530 & 751 \\
Gr2 & 534 & 781 \\
\hline
\end{tabular}

n.a., not available. or epicatechin) is located in the upper unit of the condensed pigment, whereas the pelargonidin constitutes the lower one.

Mass spectra do not allow conclusions about the position of the interflavonoid linkage. In the case of proanthocyanidins, a $\mathrm{C} 4-\mathrm{C} 8$ linkage is more likely to occur than a C4-C6 one (Haslam, 1998). Based on this and the previous pigment identification made by Fossen et al. (2004), using NMR, a C4-C8 linkage between flavanol and anthocyanin moieties has been assumed for pigments St 1 and St 2 . Thus, St 1 and St 2 were tentatively identified as catechin-(4,8)-pelargonidin 3-glucoside (d1 in Fig. 1) and epicatechin-(4,8)-pelargonidin 3-glucoside (d2 in Fig. 1), respectively. Similarly, no information to differentiate between catechin and epicatechin units can
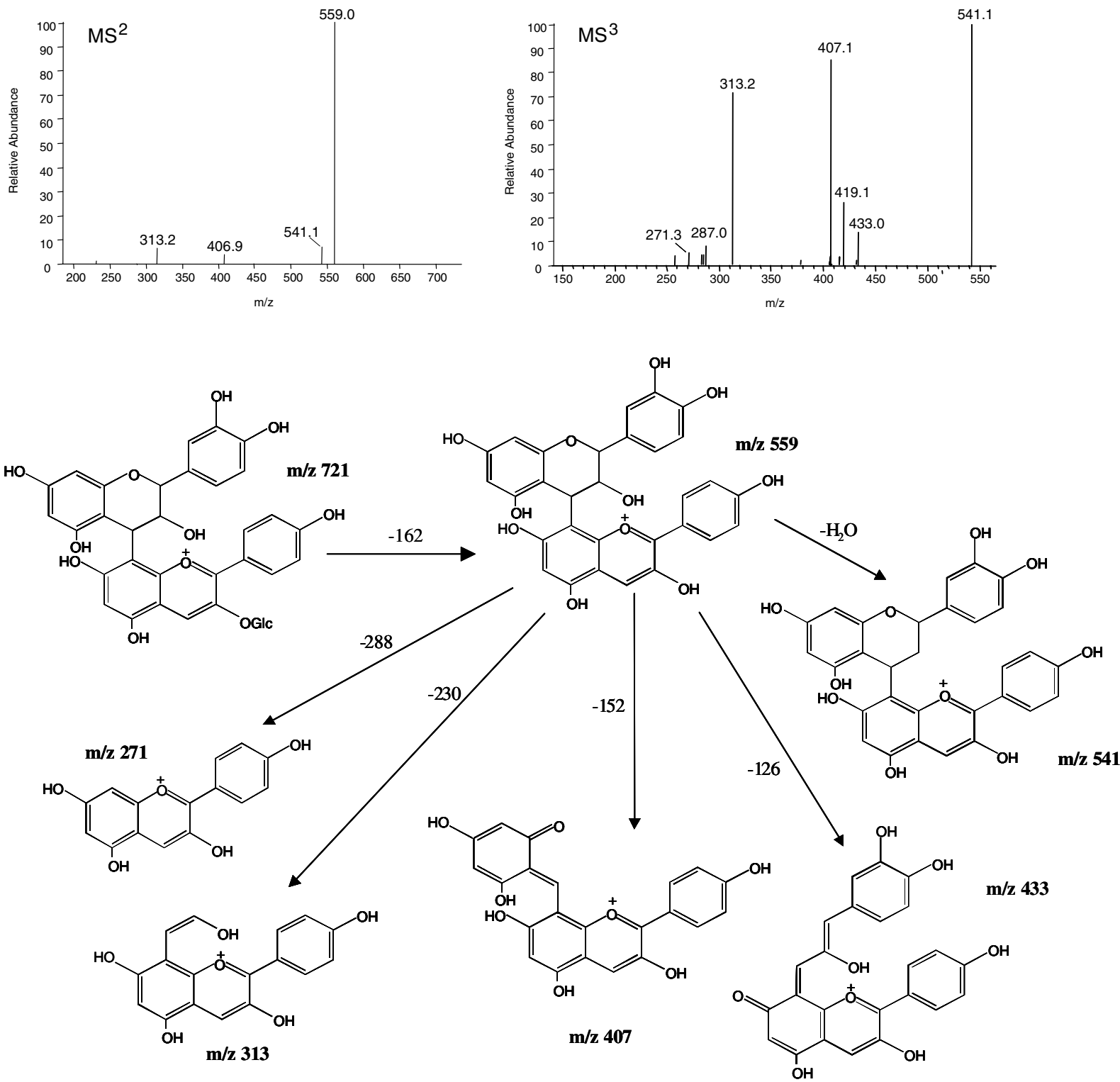

Fig. 3. $\mathrm{MS}^{2}$ and $\mathrm{MS}^{3}$ spectra of pigment St1 $(\mathrm{m} / \mathrm{z}$ 721) and scheme of fragmentation. 
be obtained from the fragmentation pattern. The assignment of the flavanol unit to pigments St1 and St2 was done according to their order of elution in the HPLC chromatogram, consistent with that observed by Fossen et al. (2004) and with the retention characteristics expected for catechins in reversed-phase columns, with $2 R, 3 S$ compounds (i.e., catechin) eluting earlier than $2 R, 3 R$ (i.e., epicatechin) (Santos-Buelga, García-Viguera, \& Tomás-Barberán, 2003). Nevetherless, it is necessary to indicate that the relative polarities of catechin and epicatechin may not be retained in all their derivatives and, in fact, the reverse order of elution was observed by Salas et al. (2004) for (epi)catechin-malvidin 3 -glucoside adducts whose formation was induced in model solutions.

Pigment St3 showed a molecular ion $[\mathrm{M}]^{+}$at $\mathrm{m} / \mathrm{z} 705$ with a similar fragmentation pattern to St1 and St2. In addition, when the molecular ion at $\mathrm{m} / \mathrm{z} 705$ was extracted from the total ion current chromatogram, the presence of a second peak (St5) was noticed, eluting at 22 min (Fig. 4). That peak was not observed in the HPLC-DAD chromatograms of the strawberry extract since it was overlapped by the peak of pelargonidin 3glucoside (major anthocyanin in strawberry). $\mathrm{MS}^{2}$ fragmentation of the molecular ion at $\mathrm{m} / \mathrm{z} 705$ yields a signal at $\mathrm{m} / \mathrm{z} 543$ (-162 amu, loss a glucose moiety) that released $\mathrm{MS}^{3}$ fragments at $\mathrm{m} / \mathrm{z} 525$ (-18 amu, loss of water), 407 (-136 amu, RDA fission of an (epi)afzelechin moiety), 313 (-230 amu, partial loss of (epi)afzelechin), and 271 (-272 amu, loss of an upper (epi)afzelechin unit). As indicated above, no conclusion can be obtained about the position of the interflavonoid linkage and the nature of the flavanol unit from the mass spectra and fragmentation pattern. As for St1 and St2, an assignment of those structural features was made, based on the identifications and elution order of the pigments previously characterised by Fossen et al. (2004). Thus, peak St 3 was tentatively identified as afzelechin$(4,8)$-pelargonidin 3-glucoside and St5 as epiafzelechin$(4,8)$-pelargonidin 3-glucoside (structures $\mathrm{d} 3$ and $\mathrm{d} 4$ in Fig. 1, respectively). Additional support for the identity of $\mathrm{St} 3$ was provided by its $\lambda_{\max }$ in the visible region (515 $\mathrm{nm}$ ), closer to that obtained by Fossen et al. (2004) for the catechin adduct $(516 \mathrm{~nm})$. Maximum wavelength of visible absorption at $520 \mathrm{~nm}$ was found by those authors for the equivalent epiafzelechin derivative, which could not be corroborated in our case due to the overlapping of St5 with the peak of pelargonidin 3-glucoside.

A molecular ion $[\mathrm{M}]^{+}$at $\mathrm{m} / \mathrm{z} 851$ was found for pigment St4, with a major $\mathrm{MS}^{2}$ fragment at $\mathrm{m} / \mathrm{z} 543$ (-308 amu, loss a rutinose moiety) that showed a $\mathrm{MS}^{3}$ fragmentation pattern identical to that of pigment St3. According to these characteristics, the peak was tentatively identified as the dimer afzelechin-(4,8)-pelargonidin 3-rutinoside (Fig. 5). The presence of afzelechin as flavanol unit and a $\mathrm{C} 4-\mathrm{C} 8$ interflavonoid linkage were assumed, based on the same considerations as for the other condensed pigments found in the strawberry extracts. There was no detection of another peak that could correspond to the epiafzelechin derivative equivalent to St4 when the total ion current chromatogram was scanned for the molecular ion $\mathrm{m} / \mathrm{z}$ 851. Such a compound, if present, would be in very small amounts,

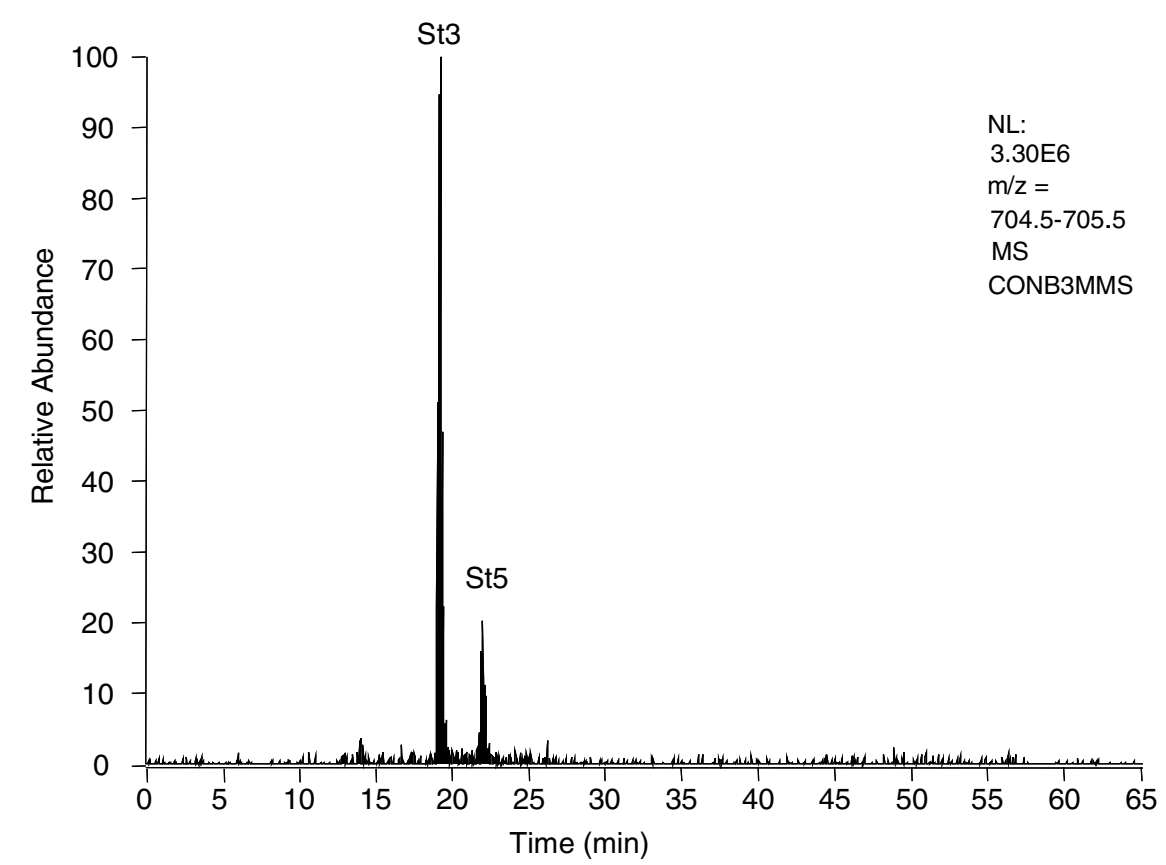

Fig. 4. Mass chromatogram for the molecular ion $\mathrm{m} / \mathrm{z} 705$ extracted from the total ion current chromatogram of the strawberry extract. 
<smiles></smiles>

Fig. 5. Structure suggested for pigment St4 detected in the strawberry.

which is not surprising taking into account the disproportion existing between the corresponding condensed pigments derived from pelargonidin 3-glucoside (i.e., $\mathrm{St} 1 / \mathrm{St} 2$ and $\mathrm{St} 3 / \mathrm{St} 5)$. Pigment St 4 was not mentioned by Fossen et al. (2004) and, according to our knowledge, it is here detected for the first time. It is notable that proportions between peaks St 3 and St 4 in the extract are similar to those existing between pelargonidin 3-glucoside and pelargonidin 3-rutinoside, the major anthocyanins in strawberry and precursors of those condensed pigments. There were no detection of peaks showing a molecular ion at $\mathrm{m} / \mathrm{z} 867$ (i.e., catechin-pelargonidin 3-rutinoside dimers).

In the anthocyanic profile of runner bean extracts (Fig. 2(b)) two minor peaks (Be1 and Be2), corresponding to condensed pigments, were detected. Peak Be1 gave a molecular ion at $\mathrm{m} / \mathrm{z} 607$ with a fragmentation scheme similar to that shown in Fig. 3. Major $\mathrm{MS}^{2}$ signals were observed at $m / z 439$ (-168 amu, RDA fission of an (epi)gallocatechin moiety), 345 (-262 amu, partial loss of (epi)gallocatechin), 589 ( $-18 \mathrm{amu}$, loss of water), 481 ( $-126 \mathrm{amu}$, loss of $\mathrm{C}_{6} \mathrm{H}_{6} \mathrm{O}_{3}$ ), and 303 (-304 amu, loss of an upper (epi)gallocatechin unit). Interestingly, no loss of a fragment corresponding to a sugar was observed, indicating that the anthocyanidin residue (cyanidin) is not glycosylated. In this respect, it is notable that major peaks detected in the chromatogram of the beans corresponded to anthocyanidins (delphinidin, cyanidin, petunidin, pelargonidin and peonidin, peaks located at 35-45 $\mathrm{min}$ in the chromatogram of Fig. 2(b)), whereas only minor peaks due to anthocyanin glycosides were detected. The presence of free anthocyanin aglycones seems to constitute a feature in the pigment composition of beans from different Phaseolus species (Macz-Pop et al., 2005). All in all, peak Bel was associated with (epi)gallocatechin-delphinidin (Fig. 6).

Peak Be2 showed a molecular ion at $\mathrm{m} / \mathrm{z} 737$ that gave a major $\mathrm{MS}^{2}$ fragment at $m / z 575(-162 \mathrm{amu}$, loss of a hexose), releasing $\mathrm{MS}^{3}$ fragment ions at $\mathrm{m} / \mathrm{z} 423$ ( $-152 \mathrm{amu}, \mathrm{RDA}$ fission of an (epi)catechin moiety), 329 (-246 amu, partial loss of an (epi)catechin unit), and $449\left(-126 \mathrm{amu}\right.$, loss of $\left.\mathrm{C}_{6} \mathrm{H}_{6} \mathrm{O}_{3}\right)$. This fragmentation allowed us to tentatively identify it as to (epi)catechin-cyanidin 3-glucoside (Fig. 6).

The pigment composition of purple corn was characterised by the presence of different anthocyanins based on cyanidin, pelargonidin and peonidin (de PascualTeresa et al., 2002). Besides them, a small peak was also observed in the HPLC chromatograms (Co1 in Fig. 2(c)) corresponding to a condensed pigment. Pigment Col had a molecular ion at $\mathrm{m} / \mathrm{z} 899$ that released two $\mathrm{MS}^{2}$ fragments at $\mathrm{m} / \mathrm{z} 737$ and 575 (successive loss of two hexose residues). The $\mathrm{MS}^{3}$ fragmentation pattern of the ion at $\mathrm{m} / \mathrm{z} 575$ was similar to that shown in Fig. 3, showing signals at $\mathrm{m} / \mathrm{z} 423$ (-152 amu, (epi)catechin RDA fission), 329 (-246 amu, partial loss of (epi)catechin), 557 ( $-18 \mathrm{amu}$, loss of water), 449 (-126 amu, loss of $\left.\mathrm{C}_{6} \mathrm{H}_{6} \mathrm{O}_{3}\right)$ and 287 (-288 amu, loss of an upper (epi)catechin unit). The appearance of two signals in the MS spectrum corresponding to the loss of two hexose residues indicated that they were located at different positions in the anthocyanidin moiety since, if they were constituting a disaccharide only one signal should have been observed, corresponding to the aglycone (Giusti, Rodríguez-Saona, Griffin, \& Wrolstad, 1999). Thus,<smiles></smiles>

Fig. 6. Structures suggested for pigments $\mathrm{Be} 1$ and $\mathrm{Be} 2$ detected in runner beans, and for pigment Co1 of purple corn. 
the pigment was tentatively identified as the condensed dimer (epi)catechin-cyanidin 3-glucoside-5-glucoside (Fig. 6). It should be noted that the precursor anthocyanin (i.e., cyanidin-3,5-diglucoside) for that pigment was not mentioned in our previous paper about pigment composition of purple corn (de Pascual-Teresa et al., 2002). However, it was further detected as a minor peak at in the chromatograms of different corn extracts (peak marked as $C y$ diglc in Fig. 2(c)).

The anthocyanin profile in grape skins of $V$. vinifera varieties has been throughly studied and it is very well known that it is based on the 3-glucosides of five anthocyanidins (delphinidin, cyanidin, petunidin, peonidin and malvidin) that may be acylated with acetic, $p$-coumaric or caffeic acids (Hebrero et al., 1988). Besides these anthocyanins, minor peaks, corresponding to flavanol-anthocyanin condensed pigments, are also usually detected in the first part of the HPLC chromatograms. Analysis of those pigments by ESI-MS revealed molecular ions and fragmentation patterns similar to that shown in Fig. 3. In Fig. 2(d) the chromatogram of a grape skin extract, where two of such pigments (marked as Gr1 and Gr2) were detected, is shown as an example. Molecular ions of pigments $\mathrm{Gr} 1$ and $\mathrm{Gr} 2$ were found at $\mathrm{m} / \mathrm{z} 751$ and 781, yielding $\mathrm{MS}^{2}$ fragments at $\mathrm{m} / \mathrm{z} 589$ and 619 (-162 amu, loss of an hexose residue), respectively. In both cases, the $\mathrm{MS}^{3}$ fragmentation was similar to that observed for the condensed pigments previously discussed, showing signals corresponding to the (epi)catechin RDA fission (-152 amu; signals at $\mathrm{m} / z 437$ and 467 for Gr1 and Gr2, respectively), partial loss of (epi)catechin (-246 amu, $m / z$ at 343 and 373), loss of water (-18 amu, $m / z$ at 571 and 601$)$, loss of $\mathrm{C}_{6} \mathrm{H}_{6} \mathrm{O}_{3}$ (-126 amu, $\mathrm{m} / \mathrm{z}$ at 463 and 493), and loss of an upper (epi)catechin unit (-288 amu, $\mathrm{m} / \mathrm{z}$ at 301 and 331). According to these mass characteristics, they were identified as (epi)catechin-peonidin 3-glucoside ( $\mathrm{Gr} 1)$ and (epi)catechin-malvidin 3-glucoside (Gr2) (Fig. 7). It is interesting to point out that the bathochromic shift in

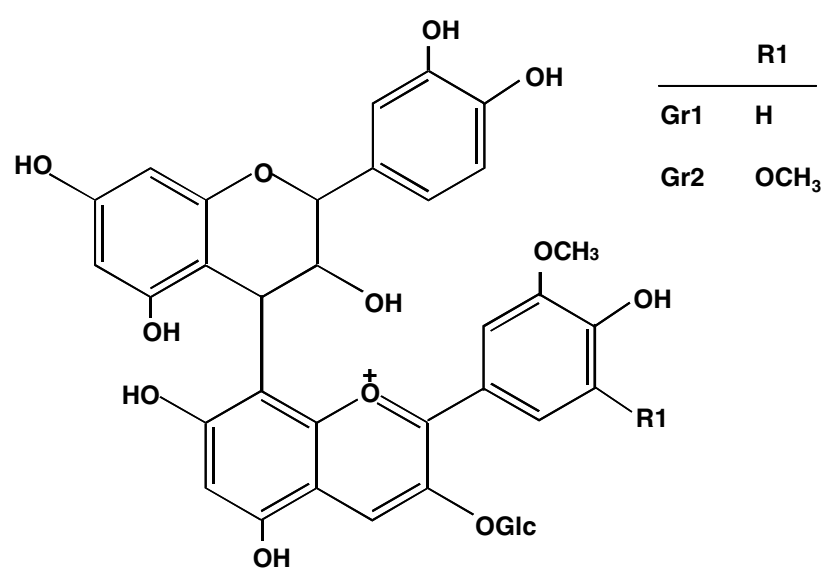

Fig. 7. Structures suggested for two flavanol-anthocyanin condensed pigments detected in grape skins. $\lambda_{\text {vis-max }}$ of pigment Gr2 $(534 \mathrm{~nm}$, Table 1) with regard to the precursor anthocyanin (malvidin 3-glucoside, $\lambda_{\max }$ at $527 \mathrm{~nm}$ in our chromatograms), was not as high as those observed for the other condensed pigments here discussed $(12-16 \mathrm{~nm})$. A similar $\lambda_{\max }(535 \mathrm{~nm})$ for the same pigment was found by Salas et al. (2004). Condensed pigments similar to Gr1 and Gr2, derived from other grape anthocyanins, have also been occasionally detected in our laboratory in the regular analyses carried out with grapes and wines (Alcalde-Eon, Escribano-Bailón, Santos-Buelga, \& Rivas-Gonzalo, 2004).

In conclusion, the results obtained suggest that flavanol-anthocyanin condensed pigments may occur in plants and they are not products exclusively formed during storage and ageing of processed food and beverages, as was previously assumed. Nevertheless, it is still possible that they may be formed in vitro during the extraction process, as a result of the reaction of the carbocations released after acid cleavage of proanthocyanidins with anthocyanins. In this respect, it is notable that, in extracts of beans obtained with water or diethyl ether without acid addition (Macz-Pop et al., 2004), the condensed pigments continued to be detected in the HPLC chromatograms in spite of a limited proanthocyanidin cleavage being expected. This observation indicates their natural presence in plants, although additional studies are necessary to obtain further confirmation.

\section{Acknowledgements}

Financial support of Junta de Castilla y León (Spain) and Spanish Comisión Interministerial of Ciencia y Tecnología through the Grants SA086/01 and AGL20020016, respectively, is greatly acknowledged. Author Lopes da Silva was funded by the European Union and Government of Portugal through the Programme PRODEP III (2001-2003).

\section{References}

Alcalde-Eon, C., Escribano-Bailón, M. T., Santos-Buelga, C., \& Rivas-Gonzalo, J. C. (2004). Separation of pyranoanthocyanins from red wine by column chromatography. Analytica Chimica Acta, 513, 305-318.

Andersen, Ø. M, Fossen, T., Torskangerpoll, K., Fossen, A., \& Hauge, U. (2004). Anthocyanin from strawberry (Fragaria ananassa) with the novel aglycone, 5-carboxypyranopelargonidin. Phytochemistry, 65, 405-410.

Bakker, J., Picinelli, A., \& Bridle, P. (1993). Model wine solutions: colour and composition changes during ageing. Vitis, 32, 111-118.

Bakker, J., Bridle, P., Honda, T., Kuwano, H., Saito, N., \& Terahara, N. (1997). Isolation and identification of a new anthocyanin occurring in some red wines. Phytochemistry, 44, 1375-1382.

Bakker, J., \& Timberlake, C. F. (1997). Isolation, identification and characterization of new color-stable anthocyanins occurring in some red wines. Journal of Agricultural and Food Chemistry, 45, $35-43$. 
Bishop, P. B., \& Nagel, C. W. (1984). Characterization of the condensation product of malvidin-3,5-diglucoside and catechin. Journal of Agricultural and Food Chemistry, 32, 1022-1026.

Dallas, C., Ricardo-da-Silva, J. M., \& Laureano, O. (1996). Interactions of oligomeric procyanidins in model wine solutions containing malvidin-3-glucoside and acetaldehyde. Journal of the Science of Food and Agriculture, 70, 493-500.

de Pascual-Teresa, S., Santos-Buelga, C., \& Rivas-Gonzalo, J. C. (2002). LC-MS of anthocyanins from purple corn cob. Journal of the Science of Food and Agriculture, 82, 1003-1006.

Es-Safi, N. E., Cheynier, V., \& Moutounet, M. (2002). Role of aldehydic derivatives in the condensation of phenolic compounds with emphasis on the sensorial properties of food-derived foods. Journal of Agricultural and Food Chemistry, 50, 5571-5585.

Fossen, T., \& Andersen, Ø. M (2003). Anthocyanins from red onion, Allium cepa, with novel aglycone. Phytochemistry, 62, 1217-1220.

Fossen, T., Rayyan, S., \& Andersen, Ø. M. (2004). Dimeric anthocyanins from strawberry (Fragaria ananassa) consisting of pelargonidin 3-glucoside covalently linked to four flavan-3-ols. Phytochemistry, 65, 1421-1428.

Francia-Aricha, E., Guerra, M., Rivas-Gonzalo, J. C., \& SantosBuelga, C. (1997). New anthocyanin pigments formed after condensation with flavanols. Journal of Agricultural and Food Chemistry, 45, 2262-2265.

Friedrich, W., Eberhardt, A., \& Galensa, R. (2000). Investigation of proanthocyanidins by HPLC with electrospray ionisation mass spectrometry. European Food Research and Technology, 211, 56-64.

Fukui, Y., Kusumi, T., Masuda, K., Iwashita, T., \& Nomoto, K. (2002). Structure of rosacyanin B, a novel pigment from the petals of Rosa hybrida. Tetrahedron Letters, 43, 2637-2639.

Fulcrand, H., Cameira dos Santos, P. J., Sarni-Manchado, P., Cheynier, V., \& Bonvin, J. F. (1996). Structure of new anthocyanin-derived wine pigments. Journal of Chemical Society and Perkin Transations, 1, 735-739.

Giusti, M., Rodríguez-Saona, L., Griffin, D., \& Wrolstad, R. (1999). Electrospray and tandem mass spectrometry as tools for anthocyanin characterization. Journal of Agricultural and Food Chemistry, 47, 4657-4664.

Haslam, E. (1998). Practical polyphenolics. From structure to molecular recognition and physiological action. Cambridge, UK: Cambridge University Press.

Hebrero, E., Santos-Buelga, C., \& Rivas-Gonzalo, J. C. (1988). HPLC-DAS identification of anthocyanins of Vitis vinifera variety Tempranillo. American Journal of Enology and Viticulture, 39, 227-234.

Heredia, F. J., Francia-Aricha, E. M., Rivas-Gonzalo, J. C., Vicario, I. M., \& Santos-Buelga, C. (1998). Chromatic characterization of anthocyanins from red grapes. I. pH effect. Food Chemistry, 63, 491-498.

Jurd, L. (1967). Catechin flavylium salt condensation reactions. Tetrahedron, 23, 1057-1064.

Jurd, L. (1969). Review of polyphenol condensation reactions and their possible occurrence in the aging of wines. American Journal of Enology and Viticulture, 20, 191-195.

Jurd, L., \& Somers, T. C. (1970). The formation of xanthylium salts from proanthocyanidins. Phytochemistry, 9, 419-427.

Liao, H., Cai, Y., \& Haslam, E. (1992). Polyphenols interactions. Anthocyanins: copigmentation and colour changes in red wines. Journal of the Science of Food and Agriculture, 59, 299-305.

Lopes da Silva, F., de Pascual-Teresa, S., Rivas-Gonzalo, J. C., \& Santos-Buelga, C. (2002). Identification of anthocyanin pigments in strawberry (cv. Camarosa) by LC using DAD and ESI-MS detection. European Food Research and Technology, 214, $248-253$.
Lu, Y., Sun, Y. S., \& Foo, L. Y. (2000). Novel pyranoanthocyanins from black currant seed. Tetrahedron Letters, 41, 5975-5978.

Macz-Pop, G.A., Rivas-Gonzalo, J.C., Pérez-Alonso, J.J., \& González-Paramás, A.M. (2005). Natural occurrence of free anthocyanin aglycones in beans (Phaseolus vulgaris L.). Food Chemistry (accepted).

Mateus, N., Pascual-Teresa, S., Rivas-Gonzalo, J., Santos-Buelga, C., $\&$ de Freitas, V. (2002). Structural diversity of anthocyanin-derived pigments in port wines. Food Chemistry, 76, 335-342.

Mateus, N., Silva, A., Santos-Buelga, C., Rivas-Gonzalo, J., \& de Freitas, V. (2002). Identification of anthocyanin-flavanol pigments in red wines by NMR and mass spectrometry. Journal of Agricultural and Food Chemistry, 50, 2110-2116.

Pissarra, J., Mateus, N., Rivas-Gonzalo, J. C., Santos-Buelga, C., \& De Freitas, V. A. P. (2003). Reaction between malvidin 3-glucoside and $(+)$-catechin in model solutions containing different aldehydes. Journal of Food Science, 68, 476-481.

Pissarra, J., Lourenço, S., González-Paramás, A. M., Mateus, N., Santos-Buelga, C., \& de Freitas, V. (2004). Formation of new anthocyanin-alkyl/aryl-flavanol pigments in model solutions. Analytica Chimica Acta, 513, 215-221.

Remy, S., Fulcrand, H., Labarbe, B., Cheynier, V., \& Moutounet, M. (2000). First confirmation in red wine of products resulting from direct anthocyanin-tannin reactions. Journal of the Science of Food and Agriculture, 80, 745-751.

Remy-Tanneau, S., Le Guernevé, C., Meudec, E., \& Cheynier, V. (2003). Characterization of a colorless anthocyanin-flavan-3-ol dimer containing both carbon-carbon and ether interflavonoid linkages by NMR and mass spectrometry. Journal of Agricultural and Food Chemistry, 51, 3592-3597.

Rivas-Gonzalo, J., Bravo-Haro, S., \& Santos-Buelga, C. (1995). Detection of compounds formed through the reaction of malvidin 3-monoglucoside and catechin in the presence of acetaldehyde. Journal of Agricultural and Food Chemistry, 43, 1444-1449.

Roggero, J. P., Coen, S., Archier, P., \& Rocheville-Divorne, C. (1987). Etude par C.L.H.P. de la reaction glucoside de malvidineacétaldéhyde-composé phenolique. Connaissance de la Vigne et du Vin, 21, 163-168.

Salas, E., Atanasova, V., Poncet-Legrand, C., Meudec, E., Mazauric, J. P., \& Cheynier, V. (2004). Demonstration of the occurrence of flavanol-anthocyanin adducts in wine and model solutions. Analytica Chimica Acta, 513, 325-332.

Santos-Buelga, C., Bravo-Haro, S., \& Rivas-Gonzalo, J. C. (1995). Interactions between catechin and malvidin-3-monglucoside in model solutions. Zeitschrift für Lebensmittel Untersuchung und Forschung, 201, 269-274.

Santos-Buelga, C., García-Viguera, C., \& Tomás-Barberán, F. A. (2003). On-line identification of flavonoids by HPLC coupled to diode array detection. In C. Santos-Buelga \& G. Williamson (Eds.), Methods in polyphenol analysis (pp. 92-127). Cambridge, UK: Royal Society of Chemistry.

Somers, T. C. (1971). The polymeric nature of wine pigments. Phytochemistry, 10, 2175-2186.

Timberlake, C. F., \& Bridle, P. (1976). Interactions between anthocyanins, phenolic compounds, and acetaldehyde and their significance in red wines. American Journal of Enology and Viticulture, 27, 97-105.

Vivar-Quintana, A. M., Santos-Buelga, C., Francia-Aricha, E., \& Rivas-Gonzalo, J. C. (1999). Formation of anthocyanin-derived pigments in experimental red wines. Food Science and Technology International, 5, 347-352.

Vivar-Quintana, A. M., Santos-Buelga, C., \& Rivas-Gonzalo, J. C. (2002). Anthocyanin-derived pigments and colour of red wines. Analytica Chimica Acta, 458, 147-155. 